\title{
The value of trefoil factor 3 expression in predicting the long-term outcome and early recurrence of colorectal cancer
}

\author{
KIYOTO MORITO $^{1}$, JUN NAKAMURA ${ }^{1}$, YOSHIHIKO KITAJIMA ${ }^{1,2}$, KEITA KAI $^{3}$, \\ TOMOKAZU TANAKA ${ }^{1}$, HIROSHI KUBO ${ }^{1}$, SHUUSUKE MIYAKE ${ }^{1}$ and HIROKAZU NOSHIRO ${ }^{1}$ \\ ${ }^{1}$ Department of Surgery, Saga University Faculty of Medicine, Saga 849-8501; \\ ${ }^{2}$ Department of Surgery, National Hospital Organization Higashisaga Hospital, Saga 849-0101; \\ ${ }^{3}$ Department of Pathology and Microbiology, Saga University Faculty of Medicine, Saga 849-8501, Japan
}

Received September 1, 2014; Accepted October 16, 2014

DOI: $10.3892 /$ ijo.2014.2755

\begin{abstract}
The trefoil factor (TFF) family comprises three thermo-stable and protease-resistant proteins (TFF1, TFF2 and TFF3) and plays an essential role in gastrointestinal mucosa protection and regeneration, and TFFs have recently been found to be involved in the development and progression of various types of cancer. However, the clinical significance of TFFs in colorectal cancer (CRC) patients remains unclear. The present study determined the relationship between TFF expression and clinicopathological findings, as well as longterm outcome in CRC patients. The mRNA expression levels of TFFs were examined in the excised CRC specimens obtained from 154 consecutive CRC patients who underwent surgical resection between 2005 and 2007 at our institution. TFF3 expression was significantly associated with the presence of distant metastasis $(\mathrm{P}=0.017)$, although neither TFF1 nor TFF2 expression was associated with the clinicopathological features. Survival rate of the patients with positive TFF3 was significantly worse compared to those with negative TFF3 $(\mathrm{P}=0.011)$. A multivariate analysis revealed that the expression of TFF3, lymph node metastasis, and vascular invasion were independent prognostic factors for disease-specific survival. Furthermore, among 134 patients with no clinical findings of metastasis at surgery, the patients with positive TFF3 experienced recurrence within one year more frequently than those with negative TFF3 $(\mathrm{P}=0.039)$. In conclusion, TFF3 is not only a useful biomarker for a long-term surgical result in CRC patient, but also may be a risk factor of early recurrence.
\end{abstract}

\section{Introduction}

Colorectal cancer (CRC) is the fourth leading cause of cancerrelated mortality worldwide, accounting for over 600,000

Correspondence to: Professor Hirokazu Noshiro, Department of Surgery, Saga University Faculty of Medicine, Nabesima 5-1-1, Saga 849-8501, Japan

E-mail: noshiro@cc.saga-u.ac.jp

Key words: trefoil factor, colorectal cancer, metastasis, survival, recurrence deaths annually (1). Although surgical resection is the most powerful treatment for the complete cure of CRC, a local or distant tumor recurrence sometimes develops even after a curative resection of the primary tumor is achieved. Therefore, the identification of reliable criteria and/or novel biomarkers for predicting a risk of recurrence is needed to identify the patients who should receive postoperative adjuvant chemotherapy. Recent clinical trials and basic research have designed to investigate biomolecules available as prognostic factors (2). However, none of them have been incorporated into the clinical practice to assess patients with CRC.

The trefoil factor (TFF) proteins (TFF1, TFF2 and TFF3) are characterized by the presence of at least one 40-aminoacid protein domain with three conserved disulfide bonds, which is termed the trefoil motif $(3,4)$. TFF1 and TFF2 are usually expressed in the stomach and duodenal epithelium, respectively. TFF3 is predominantly expressed in the goblet cells of the small intestine and colon (4). TFFs are rapidly and coordinately secreted from mucus-secreting cells when the gastrointestinal mucosa suffers mechanical and/or chemical damage, and they respond to the mucosal injury $(4,5)$. Recent studies suggest that TFFs are involved in the development and progression of various types of cancer, including breast, lung, prostate, stomach and colon cancer (6-15). In general, TFF1 seems to function as a stomach-specific tumor suppressor gene $(16,17)$, whereas TFF2 and TFF3 are thought to augment tumor progression by increasing tumor invasion and metastasis $(16,18)$. However, the more precise role of TFFs in human cancer remains unclear. There are few reports on studies that investigated the clinical significance of TFFs expression using excised primary CRC specimens (19). In the present study, we evaluated the mRNA expression levels of TFF1, TFF2 and TFF3 in excised CRC specimens and assessed the correlation between TFF expression and the clinicopathological findings in CRC patients.

\section{Materials and methods}

Patients and tissue samples. A total of 154 consecutive patients with CRC who underwent surgical resection of the primary tumor between January 2005 and December 2007 at the Saga University Hospital were enrolled in the present study. Twenty 
patients with distant metastasis who underwent a palliative resection of the tumor to release the disease-related symptoms were also included. All patients were histologically diagnosed with CRC, and none of them had received chemotherapy and/or radiotherapy before surgery. The clinicopathological findings are described according to the criteria of the TNM Classification System of Malignant Tumors 7th edition (20). Adjuvant chemotherapy was added after surgery in 47 patients. Among them, 40 patients received oral tegafur-uracil (UFT) plus leucovorin, 6 patients received $S-1$, and 1 patient received intravenous 5-fluorouracil (5-FU) with leucovorin for at least 6 months. The median follow-up period after surgery was 59.3 months (range, 2.0-88.7 months). Informed consent for the use of tissue specimens was obtained from all patients, and the study protocol was approved by the Ethics Committee of Saga University, Faculty of Medicine.

Total RNA isolation and real-time RT-PCR. At surgery, all samples of normal and cancer tissue obtained from excised specimens were immediately flash frozen in liquid nitrogen and stored at $-80^{\circ} \mathrm{C}$ until use. Total RNA was extracted from each tumor and the corresponding normal tissue specimens with the RNeasy total RNA reagent set (Qiagen, Venlo, The Netherlands). For each sample, $1 \mu \mathrm{g}$ RNA was converted into cDNA using a ReverTra Ace (Toyobo, Osaka, Japan) reverse transcription (RT) reaction kit. The cDNA was used as a template for the polymerase chain reaction (PCR). Real-time quantitative RT-PCR was performed with the LightCycler ${ }^{\mathrm{TM}}$ instrument system (Roche Diagnostics, Mannheim, Germany) using the LightCycler-FastStart DNA Master Plus SYBRGreen kit (Roche Diagnostics) according to the manufacturer's instructions. The primers were designed as follows: TFF1 sense, 5'-TTG TGG TTT TCC TGG TGT CA-3' and antisense, 5'-GGG ACG TCG ATG GTA TTA GG-3'; TFF2 sense, 5'-AGC AAG AGT CGG ATC AGT GC-3' and antisense, 5'-AGA AGC AGC ACT TCC GAG AG-3'; TFF3 sense, 5'-CAA GCA AAC AAT CCA GAG CA-3' and antisense, 5'-CTC AGG ACT CGC TTC ATG GT-3'. All experiments were carried out in triplicate, and the mean values were calculated.

Immunohistochemistry. The paraffin-embedded sections were incubated with anti-TFF3 antibody [Human TFF3 monoclonal antibody (M01), clone 3D9, 1:500; Abnova Corp., Taipei, Taiwan] overnight at $4^{\circ} \mathrm{C}$ and with the corresponding secondary antibody for $30 \mathrm{~min}$ at room temperature. The slides were then washed in phosphate-buffered saline (PBS), followed by incubation with a DAB (3,3'-diaminobenzidine) substrate kit (Nichirei Co., Tokyo, Japan). Tumors with either cytoplasmic or both cytoplasmic and membranous staining were considered to be positive for TFF3, and the level of staining was scored according to a comprehensive scoring formula previously described (21). Briefly, the intensity of staining was scored as follows: zero, no staining; 1, weak staining; 2, moderate staining; and 3, strong staining. The extent of staining was scored as follows: zero, no positively stained cells; $1,<33 \%$ of the tumor cells had positive staining; $2,33-67 \%$ positively stained tumor cells; and 3, >67\% tumor cells with positive staining. Tumors with a total score higher than six were considered to have high TFF3 expression.

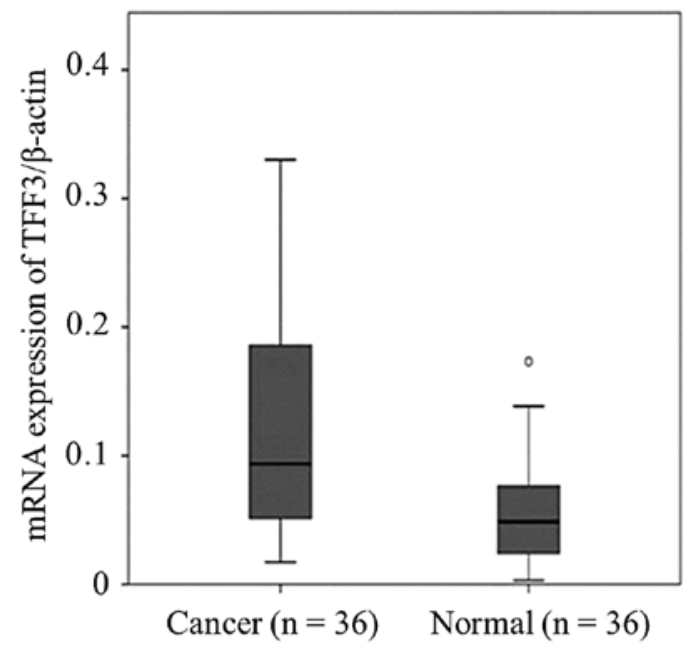

Figure 1. Box-plot shows the trefoil factor 3 (TFF3) mRNA relative expression in cancer and normal tissue obtained from colorectal cancer patients. The expression level of TFF3 in cancer tissue is significantly higher than that of the corresponding normal tissue $(\mathrm{P}=0.001)$.

Statistical analysis. Statistical analyses were performed using the computer software SPSS 15.0J for windows program (SPSS, Inc., Chicago, IL, USA). Comparisons of clinical variables between the two groups were performed with the MannWhitney U-tests. Comparisons of categorical data between the two groups were performed with Chi-square tests. A cut-off value for the TFF3 expression status was determined using a receiver-operator characteristic (ROC) curve. The survival curves were generated using the Kaplan-Meier method, and statistical differences were compared using log-rank tests. Both univariate and multivariate analyses for survival were performed using Cox's proportional hazards model. P-values $<0.05$ were considered statistically significant.

\section{Results}

Patient characteristics. The characteristics of the patients and their TFF expression levels are summarized in Table I. The 154 patients included 85 (55.2\%) males and 69 (44.8\%) females, ranging in age from 35 to 89 years (median, 69 years). Among them, 115 patients $(74.7 \%)$ were diagnosed with colon cancer, while the remaining $39(25.3 \%)$ were determined to have rectal cancer. Lymph node metastasis was found in 73 patients (47.4\%), and distant metastasis was observed in 20 patients $(13.0 \%)$.

\section{Relationship between TFF expression and the clinicopatho-} logical findings in CRC patients. The expression status of each patient's TFF was classified into a high- or low-expression group. For the purpose of the statistical analysis, the median of this series was used as a cut-off value to distinguish tumors with high or low TFF expression. As shown in Table I, neither TFF1 nor TFF2 expression was associated with the tumor histology, depth of cancer invasion, lymph node metastasis, lymphatic invasion or vascular invasion. Conversely, expression status of TFF3 was significantly associated with the location of the disease and the presence of distant metastasis $(\mathrm{P}=0.016$ and 0.017 , respectively). In addition, the expression 
Table I. Relationship between TFF family expression and clinicopathological findings.

\begin{tabular}{|c|c|c|c|c|c|c|c|c|c|}
\hline \multirow[b]{2}{*}{ Characteristics } & \multicolumn{3}{|c|}{ TFF1 expression } & \multicolumn{3}{|c|}{ TFF2 expression } & \multicolumn{3}{|c|}{ TFF3 expression } \\
\hline & $\begin{array}{c}\text { Low } \\
(n=77)\end{array}$ & $\begin{array}{l}\text { High } \\
(\mathrm{n}=77)\end{array}$ & $\mathrm{P}$-value & $\begin{array}{c}\text { Low } \\
(n=77)\end{array}$ & $\begin{array}{l}\text { High } \\
(n=77)\end{array}$ & $\mathrm{P}$-value & $\begin{array}{c}\text { Low } \\
(n=77)\end{array}$ & $\begin{array}{l}\text { High } \\
(n=77)\end{array}$ & $\mathrm{P}$-value \\
\hline Age (mean) & $68.1 \pm 10.0$ & $68.3 \pm 12.0$ & 0.924 & $66.8 \pm 11.1$ & $69.5 \pm 10.7$ & 0.129 & $67.7 \pm 10.3$ & $68.7 \pm 11.6$ & 0.583 \\
\hline \multicolumn{10}{|l|}{ Gender } \\
\hline Male & 43 & 42 & 0.871 & 43 & 42 & 0.871 & 42 & 43 & 0.871 \\
\hline Female & 34 & 35 & & 34 & 35 & & 35 & 34 & \\
\hline \multicolumn{10}{|l|}{ Location } \\
\hline Colon & 56 & 59 & 0.578 & 55 & 60 & 0.354 & 51 & 64 & 0.016 \\
\hline Rectum & 21 & 18 & & 22 & 17 & & 26 & 13 & \\
\hline \multicolumn{10}{|l|}{ Histology } \\
\hline Well & 30 & 31 & 0.869 & 34 & 27 & 0.249 & 32 & 29 & 0.621 \\
\hline Others & 47 & 46 & & 43 & 50 & & 45 & 48 & \\
\hline \multicolumn{10}{|l|}{$\mathrm{T}$} \\
\hline $1 / 2$ & 18 & 18 & 1.000 & 18 & 18 & 1.000 & 18 & 18 & 1.000 \\
\hline $3 / 4$ & 59 & 59 & & 59 & 59 & & 59 & 59 & \\
\hline \multicolumn{10}{|l|}{$\mathrm{N}$} \\
\hline Negative & 42 & 39 & 0.628 & 43 & 38 & 0.420 & 42 & 39 & 0.628 \\
\hline Positive & 35 & 38 & & 34 & 39 & & 35 & 38 & \\
\hline \multicolumn{10}{|l|}{ ly } \\
\hline Negative & 28 & 34 & 0.324 & 32 & 30 & 0.742 & 32 & 30 & 0.742 \\
\hline Positive & 49 & 43 & & 45 & 47 & & 45 & 47 & \\
\hline \multicolumn{10}{|l|}{$\mathrm{v}$} \\
\hline Negative & 52 & 52 & 1.000 & 53 & 51 & 0.731 & 51 & 53 & 0.731 \\
\hline Positive & 25 & 25 & & 24 & 26 & & 26 & 24 & \\
\hline \multicolumn{10}{|l|}{ Stage } \\
\hline 0/I/II & 41 & 36 & 0.420 & 42 & 35 & 0.259 & 42 & 35 & 0.259 \\
\hline III/IV & 36 & 41 & & 35 & 42 & & 35 & 42 & \\
\hline \multicolumn{10}{|c|}{ Distant metastasis } \\
\hline Negative & 71 & 63 & 0.055 & 68 & 66 & 0.632 & 72 & 62 & 0.017 \\
\hline Positive & 6 & 14 & & 9 & 11 & & 5 & 15 & \\
\hline
\end{tabular}

T, depth of cancer invasion; N, lymph node metastasis; ly, lymphatic invasion; v, vascular invasion. Bold P-values are statistically significant.

level of TFF3 in cancer tissue was significantly higher than that of the corresponding normal tissue $(\mathrm{P}=0.001)$ (Fig. 1).

Immunohistochemical staining for TFF3. The immunohistochemical staining for TFF3 was predominantly distributed in the cytoplasm of cancer cells. Fig. 2A and B indicates low TFF3 expression, while Fig. 2C and D depicts high TFF3 expression. The immunohistochemical staining status of TFF3 was significantly correlated with the corresponding mRNA expression level (data not shown).

Definition of the cut-off value for TFF3 expression status. The TFF3 expression status was re-classified into positive- and negative-expression groups using the appropriate cut-off value that was determined by a ROC curve generated according to the presence of distant metastasis. The cut-off value was deter- mined to be 0.134 with $60.0 \%$ sensitivity and $73.1 \%$ specificity [area under the curve (AUC) 0.662, 95\% CI 0.531-0.793, $\mathrm{P}=0.020]$. Forty-eight patients were classified as belonging to the positive expression group, while the remaining 106 patients were included in the negative expression group.

Kaplan-Meier survival analysis according to TFF3 expression. The Kaplan-Meier survival analysis with log-rank tests revealed a strong correlation between TFF3 expression and disease-specific survival (Fig. 3). The patients with positive TFF3 expression $(n=48)$ had a significantly shorter survival than those who were negative for TFF3 $(\mathrm{P}=0.011)$.

Univariate and multivariate analyses for disease-specific survival in 154 CRC patients. As summarized in Table II, tumor histology [hazard ratio $(\mathrm{HR})=2.827, \mathrm{P}=0.004)$, cancer 
Table II. Statistical analysis of disease-specific survival of colorectal patients $(n=154)$.

\begin{tabular}{|c|c|c|c|c|c|c|}
\hline \multirow[b]{2}{*}{ Characteristics } & \multicolumn{3}{|c|}{ Univariate analysis } & \multicolumn{3}{|c|}{ Multivariate analysis } \\
\hline & HR & $95 \% \mathrm{CI}$ & P-value & HR & $95 \% \mathrm{CI}$ & P-value \\
\hline Age ( $\leq 69$ vs. $>69$ years) & 0.568 & $0.076-4.237$ & 0.581 & & & \\
\hline Gender (male vs. female) & 0.767 & $0.424-1.388$ & 0.381 & & & \\
\hline Location (colon vs. rectum) & 1.419 & $0.701-2.872$ & 0.331 & & & \\
\hline Histology (well vs. others) & 2.827 & $1.401-5.705$ & 0.004 & 1.779 & $0.865-3.655$ & 0.117 \\
\hline $\mathrm{T}(1 / 2$ vs. $3 / 4)$ & 5.464 & $1.689-17.544$ & 0.005 & 0.417 & $0.112-1.209$ & 0.160 \\
\hline $\mathrm{N}$ (negative vs. positive) & 4.167 & $2.119-8.197$ & $<0.001$ & 2.681 & $1.259-5.714$ & 0.011 \\
\hline ly (negative vs. positive) & 4.255 & $1.894-9.524$ & $<0.001$ & 0.625 & $0.250-1.497$ & 0.309 \\
\hline v (negative vs. positive) & 2.825 & $1.580-5.051$ & $<0.001$ & 1.905 & $1.046-3.460$ & 0.035 \\
\hline TFF3 (negative vs. positive) & 2.103 & $1.173-3.771$ & 0.013 & 2.226 & $1.221-4.057$ & 0.009 \\
\hline
\end{tabular}

T, depth of cancer invasion; N, lymph node metastasis; ly, lymphatic invasion; v, vascular. Bold P-values are statistically significant.
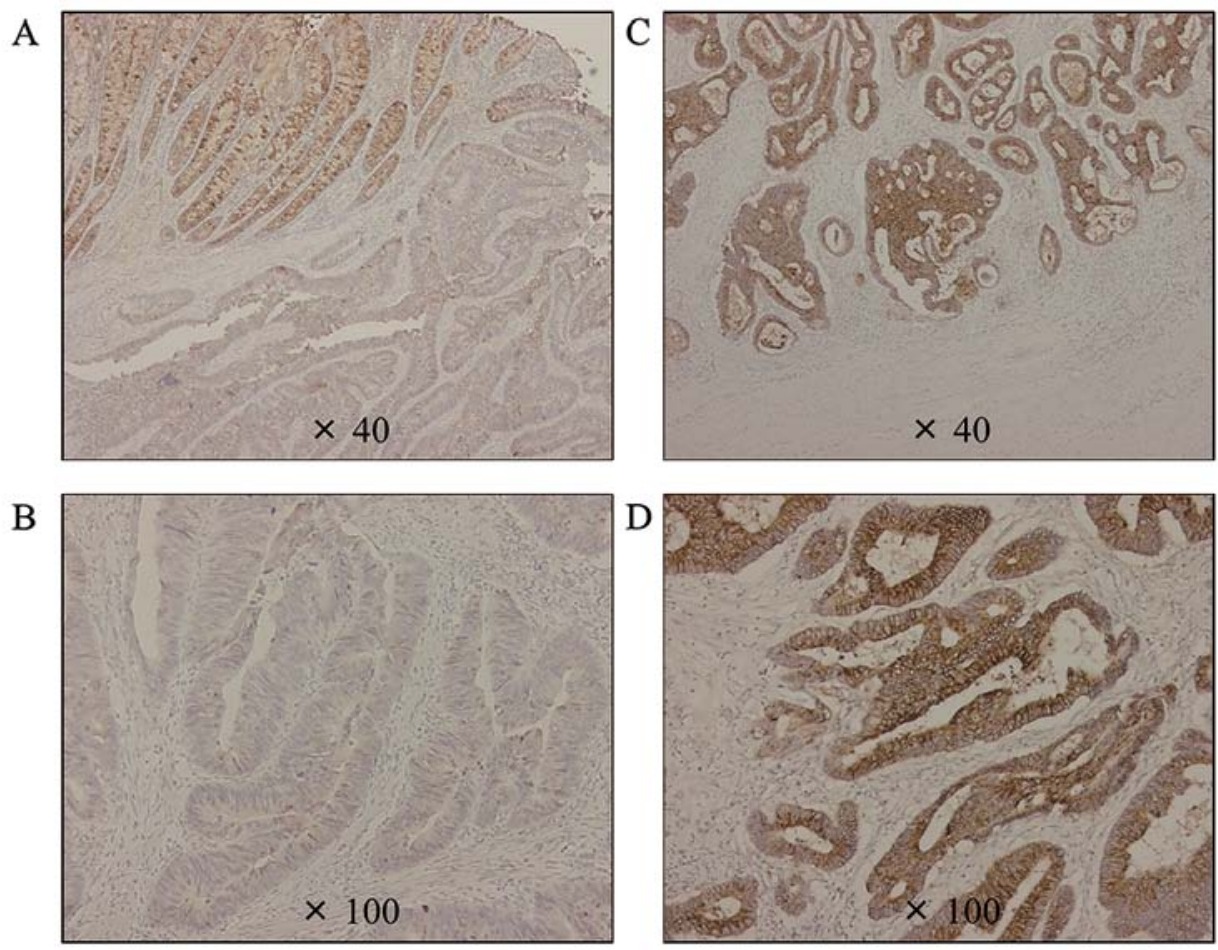

Figure 2. TFF3 expression by immunohistochemistry. (A and B) A representative case of TFF3-negative colorectal cancer (A, original magnification, x40, $\mathrm{B}$, original magnification, $\mathrm{x} 100)$. (C and D) A representative case of TFF3-positive colorectal cancer (C, original magnification, $\mathrm{x} 40$; D, original magnification, $\mathrm{x} 100)$. TFF3 staining is predominantly observed in the cytoplasm of cancer cells.

invasion depth $(\mathrm{HR}=5.464, \mathrm{P}=0.005)$, regional lymph node metastasis $(\mathrm{HR}=4.167, \mathrm{P}<0.001)$, lymphatic invasion $(\mathrm{HR}=4.255, \mathrm{P}<0.001)$, vascular invasion $(\mathrm{HR}=2.825, \mathrm{P}<0.001)$, and the positive expression of TFF3 ( $\mathrm{HR}=2.103, \mathrm{P}=0.013)$ were significantly associated with poor survival according to the univariate analysis. In addition, the multivariate analysis of the variables that were significant according to the univariate analysis was carried out using Cox's proportional hazards model (Table II). The results demonstrated that positive lymph node metastasis $(\mathrm{HR}=2.681, \mathrm{P}=0.011)$, vascular invasion $(\mathrm{HR}=1.905, \mathrm{P}=0.035)$, and the positive expression of TFF3
$(\mathrm{HR}=2.226, \mathrm{P}=0.009)$ were independently associated with poor survival in CRC patients.

The prognostic impact of TFF3 for early recurrence after curative surgery. Of the total 154 patients 134 did not have any findings of metastasis at surgery. However, 36 of the 134 patients exhibited positive TFF3 expression, and 7 of the 36 patients (19.4\%) developed recurrent disease within one year. On the contrary, local or distant tumor recurrence was observed in only 7 of 98 (7.1\%) patients with negative TFF3 expression. The frequency of early recurrence within one 


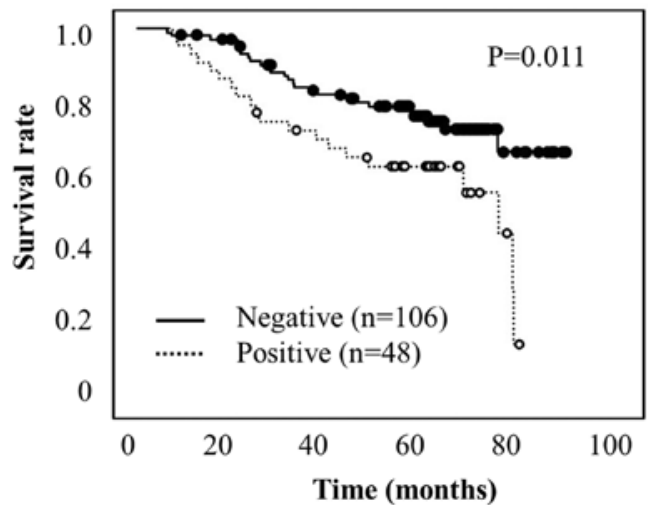

Figure 3. Disease-specific survival curves of patients with colorectal cancer according to the expression of TFF3. Patients with positive TFF3 expression exhibit significantly poorer prognosis than those with negative TFF3 expression.

year after curative surgery was significantly different between TFF3-positive and -negative patients $(\mathrm{P}=0.039)$.

\section{Discussion}

This is the first report demonstrating that positive TFF3 expression could be a prognostic marker for recurrence in CRC patients who underwent curative resection by using the excised specimens. The TFF proteins are widely expressed in the gastrointestinal epithelium, and they are well known for their protective and healing effects after mucosal damage in the gastrointestinal tract $(4,5)$. TFFs protect cells from apoptotic death and stimulate cell migration to achieve their role in epithelial tissue $(22,23)$. Although most studies have focused on their effects following mucosal injury, recent evidence has indicated that TFFs contribute to the oncogenic transformation, growth and metastasis of human solid tumors (21,24-26). However, to the best of our knowledge, the role of TFF expression in CRC has never been investigated in excised specimens. The present study evaluated the expression status of TFFs in a large cohort of samples from patients with CRC. Our results demonstrate the clinical significance of TFFs and highlight their potential as therapeutic targets in CRC patients.

TFF1 was initially described as an estrogen-inducible gene in the hormone-sensitive breast cancer cell line MCF-7 (27). However, a later observation that TFF1 knockout mice develop gastric adenomas and adenocarcinomas indicated the role of TFF1 as a tumor suppressor in the stomach (16). Our present study also demonstrated that loss of TFF1 expression in gastric cancer contributed to tumor invasion and was associated with poor survival (17). TFF1 is absent in normal colon tissue but is induced at high levels in CRCs (28). Rodrigues et al (29) demonstrated that forced TFF1 expression promotes adenomacarcinoma transition in human colon epithelial cells by using premalignant adenoma cells. In this series, TFF1 expression status was not associated with clinicopathological findings such as tumor histology, cancer invasion depth and lymph node metastasis (Table I). One previous study reported that TFF1 does not have any clinicopathological significance in CRCs (30). Taken together, the existing evidence seems to indicate that TFF1 is involved in the development of CRC rather than its progression.
The role of TFF2 in cancer biology remains unclear. Kosriwong et al (31) showed that positive TFF2 immunostaining in cholangiocarcinoma was markedly increased compared with normal and precancerous tissues, thus, suggesting that it plays an important role in tumor progression. In contrast, another study demonstrated that Helicobacter pylori infection promotes hypermethylation of the TFF2 promoter and silencing of TFF2 expression during gastric carcinogenesis, indicating that it acts as a tumor-suppressor gene (32). Therefore, the role of TFF2 in human malignancies has been controversial so far and relationship between TFF2 expression and the clinical parameters of CRCs unknown. Similarly, in our data, clinical values of TFF2 in patients with CRC are still obscure.

TFF3 is upregulated in most human malignancies, including CRC. Yio et al (12) demonstrated that TFF3 contributes to the aggressive behavior of colon cancer cells in a rat model. Rat colon cancer cells that natively express TFF3 showed enhanced migration and invasion and less apoptosis compared with a sister cell line that does not express TFF3. In cooperation with vascular endothelial growth factor (VEGF), TFF3 activates the signal transducer and activator of transcription (STAT) 3 signaling pathway, which promotes cellular invasion and tumor growth in human colon cancer (33). In the present study, high TFF3 expression was significantly associated with the presence of distant metastasis, including liver metastasis, lung metastasis, para-aortic lymph node metastasis and peritoneal dissemination (Table I), thus, indicating that TFF3 promotes the aggressive behavior of CRC. In addition, TFF3 expression was significantly higher in cancer tissues compared to the corresponding normal mucosa both at the mRNA level $(\mathrm{P}=0.001$; Fig. 1) and protein level (Fig. 2). The survival analysis demonstrated that patients with positive TFF3 expression showed significantly worse survival than those who were negative for TFF3 ( $\mathrm{P}=0.011$; Fig. 3). Furthermore, the prognostic value of TFF3 in CRC patients was identified as an independent prognostic factor in a multivariate analysis ( $\mathrm{P}=0.009$; Table II). Moreover, one recent study demonstrated a relationship between TFF3 expression and lymph node metastasis and concluded that TFF3 expression might play a role in promoting lymph node metastases of CRCs (19). Another group found that TFF3 was frequently expressed in both primary colon cancer and liver metastases but not in normal liver tissue (13). Taken together, the results suggest that TFF3 is associated with patient survival and the metastatic potential of CRC. Thus, TFF3 might be a possible prognostic marker for disease recurrence after curative surgery. We performed an additional statistical analysis that included the 134 patients without metastatic disease at the time of surgery. Among the 134 CRC patients who received curative surgery, patients with positive TFF3 expression exhibited a significantly higher recurrence frequency within one year in compared with those with negative TFF3. Although further studies of additional cohorts are needed, our results clearly demonstrate the prognostic potential of TFF3 for early CRC recurrence in a clinical setting.

In conclusion, positive TFF3 expression is an independent poor-prognostic factor in CRC patients, and may be a useful marker for early recurrence. 


\section{References}

1. Ferlay J, Parkin DM and Steliarova-Foucher E: Estimates of cancer incidence and mortality in Europe in 2008. Eur J Cancer 46: 765-781, 2010

2. Bacolod MD and Barany F: Molecular profiling of colon tumors: the search for clinically relevant biomarkers of progression, prognosis, therapeutics, and predisposition. Ann Surg Oncol 18 3694-3700, 2011.

3. Wong WM, Poulsom R and Wright NA: Trefoil peptides. Gut 44: 890-895, 1999

4. Hoffmann W and Jagla W: Cell type specific expression of secretory TFF peptides: colocalization with mucins and synthesis in the brain. Int Rev Cytol 213: 147-181, 2002.

5. Taupin D and Podolsky DK: Trefoil factors: initiators of mucosal healing. Nat Rev Mol Cell Biol 4: 721-732, 2003.

6. Poulsom R, Hanby AM, Lalani EN, Hauser F, Hoffmann W and Stamp GW: Intestinal trefoil factor (TFF 3) and pS2 (TFF 1), but not spasmolytic polypeptide (TFF 2) mRNAs are co-expressed in normal, hyperplastic, and neoplastic human breast epithelium. J Pathol 183: 30-38, 1997.

7. Buache E, Etique N, Alpy F, et al: Deficiency in trefoil factor 1 (TFF1) increases tumorigenicity of human breast cancer cells and mammary tumor development in TFF1-knockout mice. Oncogene 30: 3261-3273, 2011.

8. Qu Y, Yang Y, Ma D and Xiao W: Increased trefoil factor 3 levels in the serum of patients with three major histological subtypes of lung cancer. Oncol Rep 27: 1277-1283, 2012.

9. Bougen NM, Amiry N, Yuan Y, et al: Trefoil factor 1 suppression of E-cadherin enhances prostate carcinoma cell invasiveness and metastasis. Cancer Lett 332: 19-29, 2013.

10. Kirikoshi $\mathrm{H}$ and Katoh M: Expression of TFF1, TFF2 and TFF3 in gastric cancer. Int J Oncol 21: 655-659, 2002.

11. Leung WK, Yu J, Chan FK, et al: Expression of trefoil peptides (TFF1, TFF2, and TFF3) in gastric carcinomas, intestinal metaplasia, and non-neoplastic gastric tissues. J Pathol 197: $582-588,2002$

12. Yio X, Zhang JY, Babyatsky M, et al: Trefoil factor family-3 is associated with aggressive behavior of colon cancer cells. Clin Exp Metastasis 22: 157-165, 2005.

13. Babyatsky M, Lin J, Yio X, et al: Trefoil factor-3 expression in human colon cancer liver metastasis. Clin Exp Metastasis 26: 143-151, 2009.

14. Efstathiou JA, Noda M, Rowan A, et al: Intestinal trefoil factor controls the expression of the adenomatous polyposis colicatenin and the E-cadherin-catenin complexes in human colon carcinoma cells. Proc Natl Acad Sci USA 95: 3122-3127, 1998

15. Taupin D, Ooi K, Yeomans N and Giraud A: Conserved expression of intestinal trefoil factor in the human colonic adenoma-carcinoma sequence. Lab Invest 75: 25-32, 1996.

16. Lefebvre O, Chenard MP, Masson R, et al: Gastric mucosa abnormalities and tumorigenesis in mice lacking the $\mathrm{pS} 2$ trefoil protein. Science 274: 259-262, 1996.

17. Tanaka T, Nakamura J, Kitajima Y, et al: Loss of trefoil factor 1 is regulated by DNA methylation and is an independent predictive factor for poor survival in advanced gastric cancer. Int J Oncol 42: 894-902, 2013.
18. Tebbutt NC, Giraud AS, Inglese M, et al: Reciprocal regulation of gastrointestinal homeostasis by SHP2 and STAT-mediated trefoil gene activation in gp130 mutant mice. Nat Med 8: 1089-1097, 2002

19. Huang YG, Li YF, Wang LP and Zhang Y: Aberrant expression of trefoil factor 3 is associated with colorectal carcinoma metastasis. J Cancer Res Ther 9: 376-380, 2013.

20. Sobin LH, Gospodarowicz MK and Wittekind C: TNM Classification of Malignant Tumours. 7th Edition. WileyBlackwell, Oxford, 2009.

21. May FE and Westley BR: Expression of human intestinal trefoil factor in malignant cells and its regulation by oestrogen in breast cancer cells. J Pathol 182: 404-413, 1997.

22. Hoffmann W: Trefoil factors TFF (trefoil factor family) peptidetriggered signals promoting mucosal restitution. Cell Mol Life Sci 62: 2932-2938, 2005.

23. Thim L and May FE: Structure of mammalian trefoil factors and functional insights. Cell Mol Life Sci 62: 2956-2973, 2005.

24. Henry JA, Bennett MK, Piggott NH, Levett DL, May FE and Westley BR: Expression of the pNR-2/pS2 protein in diverse human epithelial tumours. Br J Cancer 64: 677-682, 1991.

25. Chan VY, Chan MW, Leung WK, Leung PS, Sung JJ and Chan FK: Intestinal trefoil factor promotes invasion in nontumorigenic Rat-2 fibroblast cell. Regul Pept 127: 87-94, 2005.

26. Dhar DK, Wang TC, Tabara H, et al: Expression of trefoil factor family members correlates with patient prognosis and neoangiogenesis. Clin Cancer Res 11: 6472-6478, 2005.

27. Masiakowski P, Breathnach R, Bloch J, Gannon F, Krust A and Chambon P: Cloning of cDNA sequences of hormone-regulated genes from the MCF-7 human breast cancer cell line. Nucleic Acids Res 10: 7895-7903, 1982.

28. Emami S, Rodrigues S, Rodrigue CM, et al: Trefoil factor family (TFF) peptides and cancer progression. Peptides 25: 885-898, 2004.

29. Rodrigues $\mathrm{S}$, Rodrigue $\mathrm{CM}$, Attoub $\mathrm{S}$, et al: Induction of the adenoma-carcinoma progression and Cdc25A-B phosphatases by the trefoil factor TFF1 in human colon epithelial cells. Oncogene 25: 6628-6636, 2006.

30. Vizoso FJ, Fagilde MC, Corte MD, et al: Cytosolic levels of an estrogen-induced breast cancer-associated peptide (TFF1/pS2) in colorectal cancer: clinical significance and relationship with steroid receptors. Int J Biol Markers 18: 301-310, 2003.

31. Kosriwong K, Menheniott TR, Giraud AS, Jearanaikoon P, Sripa B and Limpaiboon T: Trefoil factors: tumor progression markers and mitogens via EGFR/MAPK activation in cholangiocarcinoma. World J Gastroenterol 17: 1631-1641, 2011.

32. Peterson AJ, Menheniott TR, O'Connor L, et al: Helicobacter pylori infection promotes methylation and silencing of trefoil factor 2, leading to gastric tumor development in mice and humans. Gastroenterology 139: 2005-2017, 2010.

33. Rivat C, Rodrigues S, Bruyneel E, et al: Implication of STAT3 signaling in human colonic cancer cells during intestinal trefoil factor 3 (TFF3) - and vascular endothelial growth factormediated cellular invasion and tumor growth. Cancer Res 65: 195-202, 2005 